\title{
ATIVIDADES DE ARTETERAPIA NA REABILITAÇÃO DE USUÁRIOS DA ATENÇÃO PSICOSSOCIAL
}

\section{ARTETERAPY ACTIVITIES IN THE REHABILITATION OF PSYCHOSOCIAL ATTENTION USERS}

\section{ACTIVIDADES DE ARTETERAPIA EN LA REHABILITACIÓN DE USUARIOS DE LA ATENCIÓN PSICOSOCIAL}

\author{
Janaína Quinzen Willrich ${ }^{1}$, Dariane Lima Portela ${ }^{2}$, Renata Casarin ${ }^{3}$
}

\begin{abstract}
RESUMO
Objetivo: Este estudo objetivou conhecer as contribuições da arteterapia na reabilitação de usuários de um Centro de Atenção Psicossocial (CAPS). Método: Trata-se de uma pesquisa qualitativa realizada em 2016, por meio da aplicação de entrevista semiestruturada. Os dados foram analisados utilizando-se a técnica da análise temática. Resultados: A partir da análise dividiu-se os resultados em três categorias: desenvolvimento das oficinas terapêuticas, vínculo e participação e contribuição das oficinas terapêuticas na vida dos usuários. Observase que as oficinas terapêuticas promoveram a inclusão dos usuários e criação de vínculo, resultando em melhor qualidade de vida. Também verificou-se que os usuários demonstram afeto ao serviço e aos profissionais, e que as oficinas terapêuticas são benéficas para a saúde dos mesmos, ao passo que contribuem para bons relacionamentos familiares e sociais. Conclusão: Dessa forma a construção desse estudo proporcionou a compreensão de que a arteterapia tem um papel fundamental na vida dos usuários, contribuindo para sua reabilitação social.
\end{abstract}

Descritores: Enfermagem; Saúde mental; Serviços de Saúde Mental; Terapia pela arte.

\begin{abstract}
Objective: This study aimed to know the contributions of art therapy in the rehabilitation of users of a Psychosocial Care Center (CAPS). Method: It's a qualitative research was carried out in 2016 based on the application of semi-structured interview. Data were analyzed using thematic analysis. Results: From the analysis, the results were divided into three categories: development of therapeutic workshops, linkage and participation and contribution of therapeutic workshops in the life of users. It is observed that the therapeutic workshops promoted the inclusion of users and creation of bond, resulting in a better quality of life. It has also been shown that users show affection for service and professionals, and that therapeutic workshops are beneficial to their health, while contributing to good family and social relationships. Conclusion: Thus, the construction of this study provided the understanding that art therapy plays a fundamental role in the life of users, contributing to their social rehabilitation.

Descriptors: Nursing; Mental Health; Mental Health Services; Art Therapy.

\footnotetext{
${ }^{1}$ Doutora em Ciências pelo Programa de Pós-Graduação da Faculdade de Enfermagem da Universidade Federal de Pelotas.

${ }^{2}$ Especialista em Estratégia em Saúde da Família com ênfase em políticas públicas.

${ }^{3}$ Enfermeira. Universidade Federal de Pelotas.
} 


\section{RESUMEN}

Meta: Este estudio objetivó conocer las contribuciones de la arteterapia en la rehabilitación de usuarios de un Centro de Atención Psicosocial (CAPS). Método: Se trata de una investigación cualitativa realizada en 2016, por medio de la aplicación de entrevista semiestructurada. Los datos fueron analizados utilizando la técnica del análisis temático. Resultados: A partir del análisis se dividieron los resultados en tres categorías: desarrollo de los talleres terapéuticos, vínculo y participación y contribución de los talleres terapéuticos en la vida de los usuarios. Se observa que los talleres terapéuticos promovieron la inclusión de los usuarios y la creación de vínculo, resultando en una mejor calidad de vida. También se verificó que los usuarios demuestran afecto al servicio ya los profesionales, y que los talleres terapéuticos son beneficiosos para la salud de los mismos, mientras que contribuyen a buenas relaciones familiares y sociales. Conclusión: De esta forma la construcción de este estudio proporcionó la comprensión de que la arteterapia tiene un papel fundamental en la vida de los usuarios, contribuyendo para su rehabilitación social.

Palabras Clave: Enfermería; Salud mental; Servicios de Salud Mental; Terapia por el arte.

\section{INTRODUÇÃO}

O modelo psiquiátrico e asilar existente em nossa sociedade durante muitos anos foi sendo derrubado e novas correntes surgiram no sentido de trazer dignidade ao doente mental. ${ }^{1}$ No Brasil, na segunda metade da década de 70 surge o movimento da Reforma Psiquiátrica. Inspirando-se em modelos internacionais de mudança do modelo segregador por um que objetivasse o cuidado em liberdade, a reforma psiquiátrica conseguiu então avançar positivamente no sentido de reorientar as políticas de saúde mental do Brasil. $^{2}$

Sob essa perspectiva, foram traçadas linhas de reorientação da saúde mental e feitas deliberações de suma importância para o futuro dessa linha de cuidado. Uma delas foi na década de 90, a construção de uma rede de atenção à saúde mental. Essa rede permite a substituição progressiva de leitos psiquiátricos e a implantação dos CAPS (Centro de Atenção Psicossocial). ${ }^{2}$

O CAPS configura-se como um ponto estratégico da atenção psicossocial, ou seja, um serviço de saúde de caráter aberto e comunitário e que é substitutivo ao modelo asilar. Esse serviço é composto por uma equipe multiprofissional que atua sob um aspecto interdisciplinar prestando atendimento a indivíduos em sofrimento psíquico. $^{3}$

O objetivo do Centro de Atenção Psicossocial - CAPS é atuar no desenvolvimento da vida dos indivíduos, estar fixado em um espaço físico, mas também proporcionar o entendimento sobre território nos indivíduos, nas instituições e serviços que se entrelaçam 
com o cuidado em saúde. Deve garantir a cidadania dos usuários por meio de sua inclusão social, atuando estrategicamente na articulação dos serviços necessários para a autonomia dos indivíduos. Dentre as atividades realizadas no CAPS, estão inclusas as oficinas de arteterapia. ${ }^{3}$

Nise da Silveira foi uma precursora quando se fala em arteterapia, iniciou o trabalho nessa temática em 1956, quando instituiu a arteterapia como método terapêutico. Por meio de seu trabalho, Nise desenvolveu três princípios para o desenvolvimento das atividades, sendo: afetividade, atividade e liberdade. Assim, seu modo humanizado e reabilitador de tratar os pacientes recebeu imenso destaque. $^{4}$

Atualmente os CAPS são locais onde se pode promover atividades de reabilitação psicossocial, por meio dos trabalhos em grupo, e de arteterapia, por exemplo. Nise utilizava o método terapêutico ocupacional nas atividades em grupos a fim de que os usuários pudessem manifestar suas vivências. Sempre atentando para que as relações fossem afetivas, o ambiente acolhedor, permeados por forças autocurativas, ou seja, o afeto proporcionando condições para que as pessoas busquem uma reorganização em suas vidas. ${ }^{4}$ Por meio da arteterapia o indivíduo pode sentir-se incluído numa rede de atenção à saúde e inclusive, encontrar-se através das atividades artísticas que desenvolve. Essa estratégia contribui no sentido de auxiliar o usuário a explorar e descobrir seus sentimentos, ansiedades, medos e ideias, procurando resolver algumas questões e obter melhora em sua qualidade de vida. ${ }^{5}$

A justificativa dessa pesquisa se dá pelo interesse em relação ao assunto surgido a partir da observação, andamento e estruturação das oficinas terapêuticas no CAPS, além de perceber as contribuições das atividades terapêuticas no dia a dia dos usuários com transtorno mental. Sob essa perspectiva o presente estudo teve como questão de pesquisa: Qual a contribuição da arteterapia no tratamento de saúde mental num CAPS de uma cidade da região Sul do estado do Rio Grande do Sul? Para responder a esta questão tem-se como objetivo: conhecer a contribuição das atividades arteterapia no tratamento de usuários de um Centro de Atenção Psicossocial (CAPS).

\section{MÉTODO}

Trata-se de um estudo descritivo de abordagem qualitativa, realizada em junho de 2016, em um Centro de Atenção Psicossocial (CAPS) do tipo II, em uma cidade do sul do Rio Grande do Sul. Esta pesquisa observa os princípios éticos em pesquisas que envolvem seres humanos 
respeitando os critérios da Resolução $n^{\circ}$. 466/12 do Conselho Nacional de Saúde (CNS) que trata da pesquisa envolvendo seres humanos. ${ }^{6}$ Obedece ainda ao Código de Ética dos Profissionais de Enfermagem, embasado na Resolução do COFEN 564/2017 que diz respeito a proibições e deveres. $^{7}$ A pesquisa foi devidamente aprovada pelo Comitê de Ética em Pesquisa da Faculdade de Enfermagem da Universidade Federal de Pelotas, sob parecer $n^{\circ} 1.527 .790$ de 03 de maio de 2016.

Participaram do estudo 9 usuários, sendo 8 do sexo feminino e apenas um do sexo masculino, e os participantes tinham idade entre 30 a 70 anos. A inclusão dos participantes se deu por meio de observação durante a participação em oficinas de expressão, música e artesanato. E a partir desta observação, foram convidados então a participar deste estudo. Os participantes foram identificados pela letra "P" para cada uma das entrevistas realizadas, sendo diferenciadas pelo numero posterior à letra, de forma sequencial.

Os dados foram coletados no próprio serviço a que se refere essa pesquisa. Inicialmente foi feita a observação da dinâmica das oficinas e do serviço nas duas primeiras semanas do mês de junho, interagindo com os usuários, a fim de aproximação com a temática e com os mesmos; e posto isso, na terceira semana do mês de junho, foi feita a aplicação de uma entrevista semiestruturada, de forma individual e atentando ao sigilo das informações obtidas.

Os critérios de inclusão que levaram a escolha dos usuários selecionados foram: ser usuário do CAPS; participar regularmente das atividades do CAPS; estar comunicativo e orientado; ser maior de 18 anos de idade; permitir que a entrevista seja gravada; permitir a divulgação dos dados no meio científico. Previu-se a participação de dez usuários do serviço; no entanto, a totalidade foi definida pela saturação dos dados.

Essa ferramenta consiste no cessar da coleta de dados pelo pesquisador ao entender que novos dados não trariam mais esclarecimentos para o objeto de seu estudo. Mas, tendo em vista que a amostra ideal na pesquisa qualitativa é aquela que irá traduzir as diversas dimensões do elemento estudado e objetivando a qualidade das trocas durante o andamento da pesquisa. ${ }^{8}$

A análise dos dados ocorreu por meio da técnica de análise temática, a qual tem duas importantes funções: a verificação das questões levantadas para desenvolver a pesquisa e a subjetivação do que está implícito no conteúdo adquirido. Por uma questão de organização 
cronológica divide-se essa técnica em préanálise, exploração do material e tratamento dos dados e interpretação. ${ }^{8}$

\section{RESULTADOS E DISCUSSÃO}

A partir da análise dos dados os resultados foram divididos em três categorias denominadas: Desenvolvimento das oficinas terapêuticas, vínculo e participação e contribuição das oficinas terapêuticas na vida dos usuários.

\section{Desenvolvimento das oficinas terapêuticas}

A arteterapia consiste na realização de atividades artísticas como, por exemplo: teatro, desenho, pintura, etc. Todas as atividades tem um significado e tem um objetivo em relação ao cuidado ao doente psíquico. No desenvolver das atividades o indivíduo pode acessar possibilidades de resolver seus conflitos internos, ou seja, formas de reconstruir sua saúde mental a partir da ressignificação de novas experiências. ${ }^{9}$

As oficinas de artesanato são desenvolvidas por duas técnicas em artes e uma artesã, as quais funcionam todos os dias e turnos, enquanto o CAPS estiver aberto. São confeccionados e produzidos diversos utensílios e objetos, como tapetes, almofadas, guardanapos, pinturas, entre outros. Constatou-se que os usuários que participam desta oficina são em sua maioria, mulheres, e tem muito apreço por este espaço.

[...] participo de oficinas artesanais, confeccionando guardanapos, pinturas em tela, atualmente está trabalhando em um mosaico que será exposto no próprio CAPS. (P2)

Fazemos almofadas, guardanapo, tapete e outras coisas [...] Queria que tivesse oficinas de bordado, gosto muito. Ate estou tentando trazer bordado para ver se alguém mais tem iniciativa. (P1)

As oficinas de músicas são realizadas por uma profissional técnica em música. São realizados dois encontros semanais para ensaios, geralmente relacionadas ao ritmo gaúcho. Também são realizadas danças gauchescas, organizadas pela mesma profissional, mas em um local específico na cidade, um Centro de Tradições Gaúchas (CTG) que disponibilizou duas tardes para ensaios do grupo do CAPS.

Sabe-se que são diversas as possibilidades do ser humano se comunicar, seja por maneiras verbais, ou não verbais. ${ }^{10} \mathrm{~A}$ expressão dos usuários por meio da música, por exemplo, pode possibilitar acesso a lembranças e situações significativas em sua vida. Ademais, é visto que os indivíduos sentem-se mais autônomos, descubram habilidades para 
lidar com suas dificuldades e procurem fortalecer as relações. ${ }^{11}$

$\mathrm{Na}$ invernada eu consigo dizer minhas poesias, que eu gosto, a gente se sente artista mesmo. (P6)

No breve relato da usuária acima, constata-se que os usuários sentem-se valorizados nos momentos que tem a possibilidade de expor seu trabalho para outras pessoas.

Esse sentimento de autoestima é visto como o resultado da ressignificação das expressões antes abrigadas no interior de cada indivíduo. Consequências da arteterapia, ferramenta que proporciona a modificação subjetiva, autoconhecimento e exteriorização de sentimentos. Finalmente, a exteriorização de sentimentos pode se dar por meio de diversas formas de expressão, culminando nas oficinas terapêuticas. ${ }^{5}$

Os usuários também fazem apresentações em eventos na cidade e região. $\mathrm{O}$ evento mais conhecido é na feira nacional do doce (FENADOCE), porém também realizam apresentações anualmente na Semana Farroupilha, encontros de saúde mental como: Mental Tchê, Encontrão, entre outros.

No CAPS desta pesquisa, eram realizadas oficinas de educação física. No entanto, o professor que as realizava não faz mais parte da equipe, ocasionando a perda desta atividade. Atualmente, a artesã se disponibilizou para acompanhar os usuários ao campo de futebol, localizado próximo a Rodoviária Municipal de Pelotas para atividades físicas e de lazer, esses encontros são realizados semanalmente, em datas fixas, no turno da tarde.

Nota-se que os usuários sentem muito com a perda deste profissional no serviço, pois além de atividades físicas com orientação que eram realizadas, o mesmo realizava atividades de lazer e descontração entre os usuários, como visitas a Praia do Laranjal e idas ao Cinema.

Eu gostaria que tivesse educação física, que ainda não tem. A professora está ajudando muito, ela leva os guris pra jogar bola ali na rodoviária, e eu fico junto com ela conversando. Mas tinha que ter um professor que ai ele nos levava pra fazer caminhada, alguma atividade. (P9)

[...] também gosto de educação física, mas não temos professor. (P1)

A prática de atividades físicas adquire um papel de fundamental importância, à medida que pode ter diversos objetivos com os usuários de saúde mental. Podendo ser: promoção da educação e saúde, realização de atividades criativas, estimulação cognitiva e melhoria da qualidade de vida. ${ }^{12}$

Entende-se ainda, que tais atividades podem funcionar como um 
dispositivo inclusivo aos usuários, uma vez que desenvolve socialização, confiança e respeito entre os participantes dos grupos, o que puder ser levado para sua vida diária. É interessante que as atividades possam acontecer em ambientes públicos, fora do espaço do CAPS, para que a questão da socialização possa ser realizada também dos usuários com a comunidade. ${ }^{12}$

\section{Vínculo e participação}

Durante os trechos analisados, os usuários relatam que possuem bons vínculos com os profissionais da equipe, possibilitando a formação de um ambiente familiar.

Tenho compreensão das professoras sobre o meu problema de saúde, em casa meu marido diz que é bobagem. (P2)

Pra mim é bom, a gente conversa de tudo um pouco, conselho pra tudo que é lado, de todos desde os funcionários até os usuários. (P5)

Eu adoro, porque parece que aqui esqueço o mundo, não aparecem os problemas. Parece que aqui é a casa. (P2)

A relação estabelecida nas oficinas terapêuticas entre o oficineiro e o usuário é o que vai instaurar a função terapêutica desses espaços. Ou seja, em razão da dificuldade que os usuários de saúde mental têm em estabelecer vínculos, o responsável pelas oficinas terapêuticas tem a missão de proporcionar um ambiente acolhedor, de escuta amigável e formação de vínculos. ${ }^{10}$

Percebe-se que alguns usuários tem uma frequência diária no serviço, enquanto por outro lado, somente alguns participam em alguns turnos.

[...] venho todas as tardes, já há três anos. (P3)

To com a música que é quatro dias da semana e mais as oficinas, venho praticamente todos os dias. (P6)

Eu venho com a professora de música terça e quintas, segunda e sexta na música e futebol quarta de tarde e terça de manhã tem educação fisica. $(P 7)$

A frequência e participação dos usuários nas oficinas terapêuticas deve ser de acordo com a vontade do usuário, assim como não deve haver nenhuma classificação para participar de uma ou outra oficina. Independente do seu sofrimento psíquico, idade ou gênero, o indivíduo pode optar por sua participação na oficina que lhe agradar. Tendo em vista que definir ou classificar usuário para cada oficina acaba segregando o cuidado, tornando-o estigmatizado e excludente. ${ }^{13}$

Ainda de acordo com a autora acima $^{13}$, é importante que o centro de atenção psicossocial não seja um local exclusivo de socialização dos usuários de 
saúde mental. É saudável que busquem outros locais para sua reinserção no meio social, como passeios, idas ao cinema, festas, entre outros.

Sabendo que a realidade dos usuários de saúde mental é saturada por situações excludentes e de estigmatização, a atenção psicossocial tem o intuito de redirecionar as práticas de cuidado. Devolver a esses indivíduos o sentimento de pertencimento, valorização e liberdade tem de ser o foco das atividades terapêuticas em saúde mental. ${ }^{5}$

E esse sentimento é constatado pelos usuários do serviço, quando se referem a como se sentem quando participam das oficinas terapêuticas, como podemos ver a seguir.

Eu me sinto mais livre [...] A gente conversa, vai fazendo e conversando. (P9)

O usuário de saúde mental precisa de um acompanhamento terapêutico dentro de suas singularidades, tendo em vista que cada indivíduo tem suas necessidades distintas. Cabem aos profissionais acompanhar, e estarem atentos a possíveis necessidades dos mesmos, principalmente em relação à realização de tarefas.

Espero que continue pra alegrar as pessoas. Nas oficinas a gente se sente que ainda é capaz de fazer alguma coisa. (P6)
É importante sempre destacar os pontos positivos de cada indivíduo, e mostrar que as oficinas terapêuticas podem viabilizar sua criatividade. $\mathrm{O}$ compartilhamento de espaços, a valorização de cada participante, a atenção às subjetividades no processo de criar, e a liberdade individual constatam que o modelo psicossocial do cuidado pode superar o sofrimento psíquico de cada usuário dos serviços de saúde mental. ${ }^{10}$

Desse modo, é fundamental que os profissionais do serviço saibam orientar, esclarecer, facilitar e apoiar o usuário e a família para assim promover o vínculo e a confiança na rede de cuidado.

\section{Contribuição das oficinas terapêuticas na vida dos usuários}

Ao inserir-se no Centro de Atenção Psicossocial (CAPS) o indivíduo que está em algum tipo de sofrimento psíquico recebe um acolhimento e junto da equipe de profissionais do serviço irá construir o seu Projeto Terapêutico Singular (PTS), o qual irá delinear o processo de cuidado e estabelecer estratégias com o intuito de melhorar sua qualidade de vida. ${ }^{10}$

As atividades terapêuticas do CAPS objetivam reabilitar e incluir os usuários, seja por meio de atividades de cultura, lazer ou trabalho. $\mathrm{O}$ ambiente de cuidado deve ser estimulador, respeitando as 
individualidades, promovendo a diminuição dos estigmas sociais e incentivando as potencialidades do usuário. $^{14}$

A ocorrência das oficinas terapêuticas pode ser no serviço ou também em locais da comunidade adstrita, incentivando assim os usuários a vincularem-se a outros espaços da comunidade. Podendo também ocorrer com grupos de outras comunidades a fim de enriquecer as trocas e vivências dos grupos. ${ }^{14}$

As oficinas terapêuticas configuram-se como ferramentas que podem auxiliar o usuário a trabalhar seus pensamentos e produzir algo que possa ser válido para si. Podendo levar esse usuário a um processo de reabilitação eficaz, em que ele entenda os objetivos do serviço em sua trajetória. ${ }^{10}$

A participação em oficinas é mencionada por um participante do estudo como benéfica para saúde, pois é espaço de diálogo e vínculos entre pessoas, como se pode constatar a seguir:

Acho que me ajuda muito na saúde, não tanto a arte, mas sim a conversa que temos com outras pessoas, a gente cria vínculo com as professoras e os outros pacientes. (PI)

Alguns pesquisadores ${ }^{10}$ acreditam que os espaços compartilhados de cuidado são fundamentais para a expressão das potencialidades dos indivíduos. Tendo em vista que nesses momentos os usuários interagem, há reciprocidade, e formação de vínculos com os demais participantes e profissionais do serviço.

Por meio das propostas simples da arteterapia busca-se um efeito psicoterápico na vida dos usuários. Todas as atividades são imbuídas de significado, sejam elas pintura, desenho, teatro, entre outras, pois partem da premissa de que a saúde e a criatividade são muito próximas no processo de desenvolvimento humano. ${ }^{9}$

Percebe- se que as oficinas não são somente consideradas um espaço de aprendizado e desenvolvimento de técnicas, mas também um espaço de diálogo, interação e lazer entre os usuários, o que pode ser visto a seguir nos trechos das entrevistas:

[...] a gente aprende um monte de coisa, também a gente está junto com os colegas, faz amizade boa. (P6)

Eu gosto, me ajuda. Pelo menos estou no meio do pessoal, pelo menos não estou sozinha. Eu em casa me sinto sozinha e aqui não [...]. (P4)

Começo a ver que o CAPS me ajudou [...] não pelo sinônimo de liberdade que o CAPS nos proporciona, mas por me encontrar mais nas atividades, fazer alguma coisa que eu consiga espairecer um pouco, aqui tenho com quem conversar um pouco mais abertamente. (P8) 
Sob a perspectiva dos participantes desta pesquisa, as oficinas são um espaço de convivência, que oportunizam interagir com outros usuários, além de sentirem-se produtivos.

Nesse sentido, ressalta-se que por meio da arteterapia os indivíduos conseguem intensificar seu conhecimento sobre o mundo. Do mesmo modo que podem desenvolver sua autonomia criativa, podendo modificar subjetivamente seus processos internos de sofrimento mental durante os encontros terapêuticos. ${ }^{5}$

No entanto, podemos mencionar que nem sempre o que aproxima os usuários das oficinas são as atividades propostas, mas sim a ajuda mútua entre eles, um lugar disponível para conversar e relaxar, aliviando as tensões do dia-a-dia.

Não acho motivo para viver, aqui eu esqueço isso, fico feliz, satisfeita [...]. (P2)

Senti melhor pra faze as atividades, no hospital eu ficava nervosa, aqui fico calma, tem bastante atividade. O que é bom aqui, a gente é companheiro um do outro. (P8)

Neste estudo, alguns usuários descreveram a arteterapia como a construção do ciclo de reabilitação, aprendem a respeitar as diferenças de outros usuários e familiares, possibilitando a convivência intergrupal. Do mesmo modo, outros autores ${ }^{15}$ acreditam que a relação da arte com a saúde mental é extremamente produtiva haja vista sua potencialidade de criar espaços existenciais nos indivíduos. Dessa forma, pode promover sua saúde mental a partir de outra experiência do cuidado, a arte.

Levanta-se também um questionamento a respeito dessa temática ao discutir sob a perspectiva de que é preciso avaliar rotineiramente a conexão das atividades realizadas com o dia-a-dia dos usuários em relação aos aspectos isolacionistas do sofrimento psíquico. Ademais, é fundamental que os protagonistas nesse processo de cuidado sejam os próprios usuários, acessando seus territórios existenciais e desenvolvendo questões das relações humanas, seja com o grupo, suas relações familiares e relações com outrem. ${ }^{16}$

Abaixo se observa um trecho em que o conteúdo temático aborda dificuldades de relações pessoais, as quais, com a ajuda da arteterapia, estão sendo mais bem desenvolvidas.

Ser mais paciente, conversar um pouco com as pessoas. Tem um pouco de relação com o grupo, eu não tinha muito quando era pequena, tinha dificuldade no colégio. Comecei a aprender aqui no CAPS. (P9)

$O$ jeito que elas te tratam aqui, para o comportamento da gente é muito bom, por causa, 
que a gente não fica agressivo, em casa eu consigo conversar com a mãe, ameniza tudo né. (P8)

Dado o exposto, percebe-se que as oficinas terapêuticas contribuem positivamente na vida dos usuários do referido serviço, tendo em vista que os mesmo relataram situações benéficas dos encontros em suas vidas e em sua reabilitação psíquica.

\section{CONSIDERAÇÕES FINAIS}

Com o desenvolvimento desse estudo buscou-se conhecer a contribuição das oficinas de arteterapia, desenvolvidas no CAPS, no tratamento dos usuários do serviço. Foi possível identificar então, que as oficinas terapêuticas têm como propósito a reabilitação e reinserção social, além de promover a inclusão dos usuários no serviço, criando vínculo com demais usuários e profissionais, ocasionando aos usuários melhor qualidade de vida.

Pode-se destacar que os usuários demonstram afeto ao serviço e aos profissionais, demonstrando o vínculo estabelecido, demonstrando que o suporte que recebem é fundamental em suas vidas para sua reinserção e reestabelecimento social. Acredita-se que os profissionais das equipes devem estabelecer um relacionamento construído diariamente, embasando-se na confiança com os usuários do serviço, para que o cuidado possa ser implementado da melhor forma possível.

Observou-se neste estudo, que os usuários mencionam as oficinas terapêuticas como algo benéfico para sua saúde no dia-a-dia, sendo algo essencial, que pode contribuir para relacionamentos familiares, amizades, e proporcionar uma nova forma de se relacionar com outras pessoas. Sendo assim, acredita-se que as oficinas terapêuticas são atividades que possibilitam o crescimento pessoal e social do usuário de saúde mental. Ajuda a promover a saúde e o bem-estar, ao ponto que utiliza o potencial do próprio individuo para sanar suas demandas pessoais e sociais.

Neste sentido, corrobora-se a importância dos espaços terapêuticos no processo de reabilitação dos usuários de serviços de saúde mental, fazendo com que estes espaços sejam dinâmicos e proporcionem trocas e reflexões na vida dos usuários.

Ressalva-se que com este estudo não se intenta generalizar dados aqui encontrados, uma vez que se trata de um serviço específico. Não se busca ainda o esgotamento da discussão sobre esse tema, tendo em vista a importância das oficinas terapêuticas na atenção psicossocial. 


\section{REFERÊNCIAS}

1 Desviat M, Ribeiro V. A reforma psiquiátrica. 2ed. Rio de Janeiro: FIOCRUZ; 2015.

2 Cardoso AJC, Trino A, Pedra M, Medeiros PP. Reforma psiquiátrica e a política nacional de saúde mental. Tempus (Brasília). 2014; 8(1):57-63.

3 Ministério da Saúde (Brasil). Centros de atenção psicossocial e unidades de acolhimento como lugares da atenção psicossocial nos territórios: orientações para elaboração de projetos de construção, reforma e ampliação de CAPS e de UA. Brasília, DF: Ministério da Saúde; 2015. 4 Oliveira PF, Melo Junior W, Vieira-Silva M. Afetividade, liberdade e atividade: $o$ tripé terapêutico de Nise da Silveira no Núcleo de Criação e Pesquisa Sapos e Afogados. Pesqui Prát Psicossociais. 2017; 12(1):23-35.

5 Azevedo EB, Costa LFP, Espínola LL, Silva PMC, Musse JO, Ferreira Filha, MO. Arteterapia como promotora da qualidade de vida e inclusão social de profissionais e usuários. Rev Univ Vale do Rio Verde. 2014; 12(2):167-76.

6 Conselho Nacional de Saúde (Brasil). Resolução no 466, de 12 de dezembro de 2012. Diretrizes e normas regulamentadoras de pesquisa envolvendo seres humanos [Internet]. Brasília, DF: CNS; 2012 [citado em 16 mar 2018]. Disponível em:

http://bvsms.saude.gov.br/bvs/saudelegis/c ns/2013/res0466_12_12_2012.html.

7 Conselho Federal de Enfermagem (Brasil). Resolução COFEN no 564 de 2017. Aprova a reformulação do Código de Ética dos Profissionais de Enfermagem [Internet]. Brasília, DF: COFEN; 2017 [citado em 16 mar 2018]. Disponível em: http://www.cofen.gov.br/resolucao-cofenno-5642017_59145.html

8 Minayo, MCS, organizadora. Pesquisa Social: teoria, método e criatividade. 28ed. Petrópolis, RJ: Vozes; 2009.
9 Andrade EP, Sousa PA, Andrade EP.

Arteterapia, CAPS e psicologia: relações e possibilidades de trabalho. In: $11^{\circ}$

Encontro Internacional de Formação de Professores; $12^{\circ}$ Fórum Permanente de Inovação Educacional; $4^{\circ}$ Encontro Estadual da Associação Nacional pela Formação dos Profissionais da Educação [Internet]; 2016 [citado em 14 mar 2018]; Aracajú. Aracajú: ITP; 2016. p. 1-12.

Disponível em: https://eventos.set.edu.br/index.php/enfope /article/view/1903/585

10 Farias ID, Thofehrn MB, Andrade APM, Carvalho LA, Fernandes HN, Porto AR. Oficina terapêutica como expressão da subjetividade. SMAD, Rev. Eletrônica Saúde Mental Álcool Drog. [Internet]. 2016 [citado em 14 mar 2018]; 12(3):14753.

Disponível em:

https://www.revistas.usp.br/smad/article/vi ew/120779/117846

11 Batista EC, Ferreira DF. A música como instrumento de reinserção social na saúde mental: um relato de experiência. Rev Psicol Foco [Internet]. 2015 [citado 15 mar 2018]; 7(9):67-79. Disponível em: http://revistas.fw.uri.br/index.php/psicol ogiaemfoco/article/view/1593

12 Costa e Silva TM, Oliveira HLR, Silva RCB, Santos FM, Graup S. Reflexões sobre a atuação do profissional de educação física nos centros de atenção psicossocial. Rev Perspect Ciênc Saúde [Internet]. 2017 [citado em 18 mar 2018]; 2(95):95-106. Disponível em:

http://sys.facos.edu.br/ojs/index.php/perspe ctiva/article/view/106

13 Dias JDS. Oficinas terapêuticas como estratégia para reinserção psicossocial e produção de vínculo. Pretextos [Internet] 2018 [citado em 16 mar 2018]; 3(5):12945. Disponível em: http://periodicos.pucminas.br/index.php/pr etextos/article/view/15933

14 Dutra VFD, Baptista J, Acioli L, Rapouso F, Boeira S, Oliveira RMP. A importância do ambiente terapêutico no 
cuidado em um centro de atenção psicossocial. Rev Enferm UFPE on line [Internet]. 2016 [citado em 16 mar 2018]; 10(11):4711-9. Disponível em:

https://periodicos.ufpe.br/revistas/revistaen fermagem/article/view/11248 15 Moro LM, Guazina FMN. Arte e experiência: relações da arte no contexto da saúde mental. Cad Bras Saúde Mental [Internet]. 2016 [citado em 15 mar 2018]; 8(18):25-42. Disponível em:

http://incubadora.periodicos.ufsc.br/index. php/cbsm/article/view/4027
16 Kinker FS, Imbrizi JM. O Mito das oficinas terapêuticas. Rev Polis Psique [Internet]. 2015 [citado em 16 mar 2018]; 5(3):61-79. Disponível em: http://pepsic.bvsalud.org/pdf/rpps/v5n3/ n5a05.pdf

RECEBIDO: 05/08/2018

APROVADO: 31/10/2018

PUBLICADO: 12/2018 